\title{
ARTICLE \\ Resonant magnetoelastic microstructures for wireless actuation of liquid flow on 3D surfaces and use in glaucoma drainage implants
}

\author{
Venkatram Pepakayala ${ }^{1}$, Joshua Stein ${ }^{2}$ and Yogesh Gianchandani ${ }^{1}$
}

Magnetoelastic resonators made from metal alloy foils are widely used for miniature wireless anti-theft tags and have also been explored for use in various sensing applications. Through annealing within three-dimensional (3D) molds, these foils can be formed into curved structures. Consequently, magnetoelastic materials present an opportunity for the development of a new class of wireless, actuators that have small form factors and low surface profiles and that can conform to curved surfaces. This paper describes passive, wireless, resonant magnetoelastic actuators intended for the generation of fluid flow on the surfaces of implantable Ahmed glaucoma drainage devices. The actuators are remotely excited to resonance using a magnetic field generated by external coils. The fluid flow is intended to limit cellular adhesion to the surface of the implant, as this adhesion can ultimately lead to implant encapsulation and failure. The actuators are micromachined from planar 29- $\mu$ m-thick foils of Metglas $2826 \mathrm{MB}$ $\left(\mathrm{Fe}_{40} \mathrm{Ni}_{38} \mathrm{Mo}_{4} \mathrm{~B}_{18}\right)$, an amorphous magnetoelastic alloy, using photochemical machining. Measuring $10.3 \times 5.6 \mathrm{~mm}{ }^{2}$, the planar structures are annealed in 3D molds to conform to the surface of the drainage device, which has an aspherical curvature. Six actuator designs are described, with varying shapes and resonant mode shapes. The resonant frequencies for the different designs vary from $520 \mathrm{~Hz}$ to $4.7 \mathrm{kHz}$. Flow velocities of up to $266 \mu \mathrm{m} \mathrm{s}^{-1}$ are recorded at a wireless activation range of $25-30 \mathrm{~mm}$, with peak actuator vibration amplitudes of $1.5 \mu \mathrm{m}$. Integrated actuators such as those described here have the potential to greatly enhance the effectiveness of glaucoma drainage devices at lowering eye pressure and may also be useful in other areas of medicine.

Keywords: actuator; fibrosis; glaucoma; magnetostriction; Metglas

Microsystems \& Nanoengineering (2015) 1, 15032; doi:10.1038/micronano.2015.32; Published online: 7 December 2015

\section{INTRODUCTION}

Magnetoelastic devices have wide appeal for use in applications that demand passive, wireless operation. Resonant magnetoelastic devices are commonly used in anti-theft tags ${ }^{1}$. Sensors targeting measurements of mass loading, viscosity, fluid flow, pressure, strain, and other physical parameters have been described ${ }^{2-7}$. Linear and rotary actuators have also been reported ${ }^{8,9}$, as have passive sensors that detect occlusions in biliary and peripheral artery stents ${ }^{10,11}$. Although most magnetoelastic devices are of the order of $1 \mathrm{~cm}$ in size in at least one dimension, tags as small as $1 \mathrm{~mm}$ have been reported ${ }^{12,13}$, demonstrating the degree of miniaturization that is possible with this technology.

Whereas the vast majority of reported magnetoelastic devices have been planar, a few curved structures have been described for use in sensing applications ${ }^{10,14,15}$. These three-dimensional (3D), out-of-plane geometries have been achieved by thermal annealing or by inducing elastic or plastic deformations. However, until now, these geometries have been limited to simple, singleaxis curvatures. For instance, sensors reported for detecting occlusions in biliary stents had tubular curvatures to match stent geometry ${ }^{10}$.

Glaucoma is the second leading cause of blindness globally and is expected to afflict 79.6 million people by $2020^{16,17}$. It is commonly associated with high intraocular pressure (IOP), which results from increased resistance to the outflow of aqueous humor $(\mathrm{AH})$ from inside the eye; this causes damage to the optic nerve and an irreversible loss of vision. High IOP is typically managed by pharmaceutical treatments or surgical interventions performed by laser or incision ${ }^{18}$. The pharmaceutical treatments are typically administered as eye drops ${ }^{19}$, whereas the surgical methods include laser trabeculoplasty and, to a lesser extent, glaucoma filtration surgeries ${ }^{20,21}$. Glaucoma drainage devices (GDDs) are used for cases of intractable glaucoma, although findings from recent clinical trials support the use of these devices much earlier in the disease course ${ }^{22}$. As a result, the utilization of GDDs has increased considerably over the past decade.

The Ahmed glaucoma drainage device (AGDD) uses a drainage tube that permits the flow of fluid out of the eye when the IOP exceeds a certain threshold. The tube drains $\mathrm{AH}$ into the space created by the plate body, located in a subconjunctival space over the sclera (Figure 1a). Over time, a fibrous capsule of tissue, or "bleb," is formed around the AGDD, serving as a reservoir for the extra $\mathrm{AH}$ that drains from the eye $\mathrm{e}^{23-29}$. In a well-functioning implant with a controlled IOP, the bleb has a thickness of less than $500 \mu \mathrm{m}^{24}$. However, in a subset of patients, a thick, dense inner layer of collagen forms around the plate body, reducing permeability to $A H$, which, in turn, increases the resistance to $A H$ outflow $^{30,31}$ (Figure 1b). The formation of the fibrous bleb is mediated by the adhesion and proliferation of fibroblasts and vascular endothelial cells on the implant surface. Hence, the

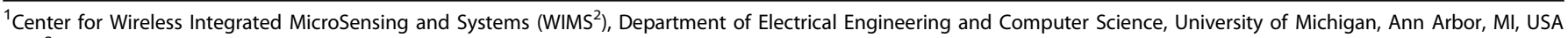
and ${ }^{2}$ Department of Ophthalmology and Visual Sciences, University of Michigan, Ann Arbor, MI, USA

Correspondence: Venkatram Pepakayala (venkatp@umich.edu)

Received: 16 June 2015; revised: 11 September 2015; accepted: 12 September 2015
} 
a

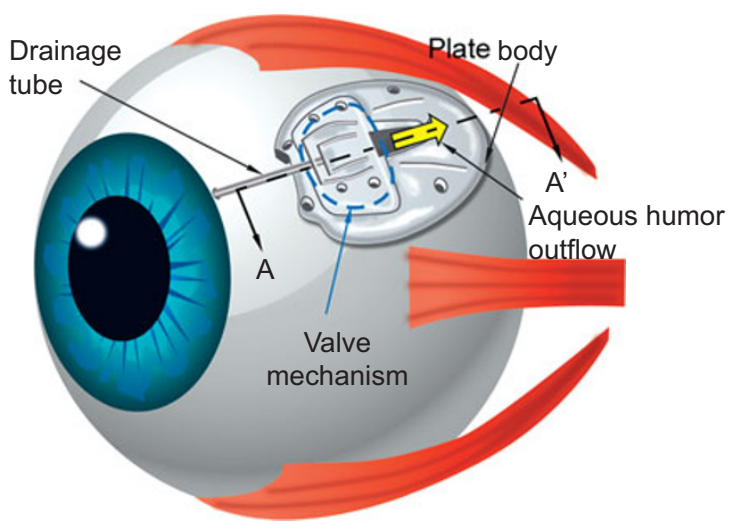

b

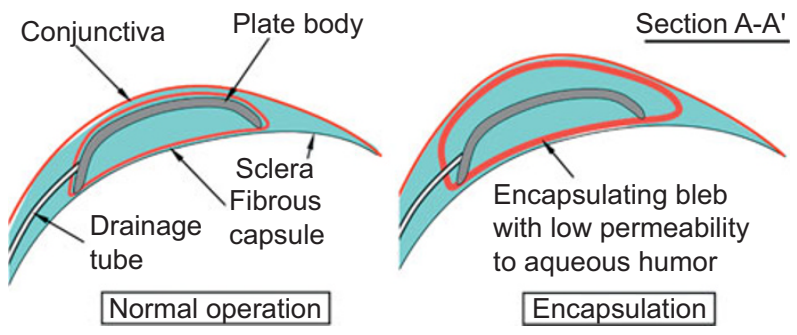

C

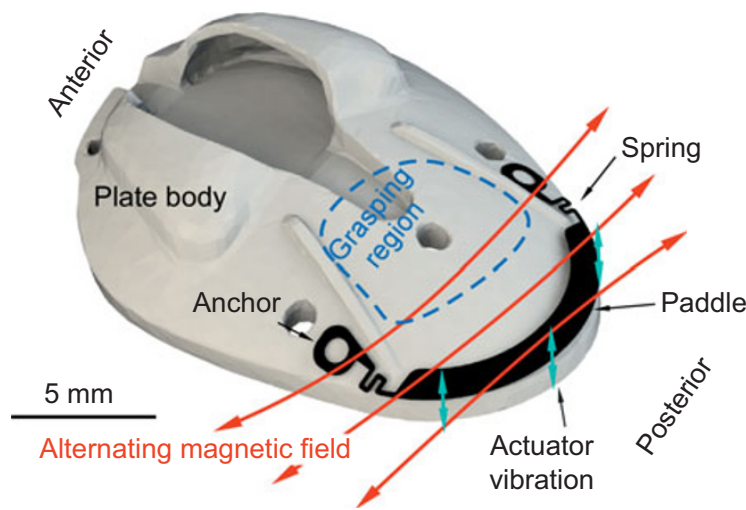

Figure 1 (a) Implantation of the Ahmed glaucoma drainage device (AGDD). The conjunctiva is not shown for clarity. (b) Cross section of the implant showing the formation of the bleb, which serves as a reservoir for aqueous humor $(\mathrm{AH})$ exiting the eyeball. As pictured on the right, a thick, encapsulated bleb with low permeability to $\mathrm{AH}$ results in the inability of the device to drain $\mathrm{AH}$ as intended, leading to an elevated IOP. (c) A magnetoelastic actuator (Type A) attached to the plate body.

extent of this fibrovascular tissue response can be correlated to the initial adhesion of these cells to the implant surface ${ }^{31,32}$

Chemical and physical surface modification measures such as plasma treatment, deposition of bioactive coatings, and texturing, among other methods, have shown promise in improving tissue integration with implantable biomaterials ${ }^{33-37}$. However, these measures are ineffective at limiting fibrous encapsulation ${ }^{33,37,38}$. Surface microstructures have been shown to lower the fibrous response to certain biomaterials ${ }^{39,40}$. Conversely, greater surface roughness has been associated with increased fibrous encapsulation in $\mathrm{GDDs}^{31,32}$, calling into question the feasibility of using this approach for GDDs. Other efforts targeted at reducing encapsulation in GDDs have been focused on the use of anti-fibrotic agents such as mitomycin C, 5-fluorouracil, and triamcinolone acetonide, but these interventions have also met with limited success $^{41-43}$. In addition, these measures can lead to complications such as necrosis of the conjunctival tissue or can contribute to an increased risk of complications such as hypotony (i.e., low IOP) or serious infection ${ }^{30,44}$.

In vitro studies have shown that local vibrations and fluid agitation that generate shear stresses can influence the adhesion and proliferation of cells $s^{45-47}$. Above a specific oscillating stress limit, human fibroblasts fail to adhere to the substrate ${ }^{45}$. Additionally, in vitro studies have also shown that vibrations negatively influence the proliferation of certain types of mouse embryonic fibroblasts ${ }^{46}$, with vibration amplitudes measuring approximately $100 \mathrm{~nm}$ at frequencies of $100 \mathrm{~Hz}$ and $1 \mathrm{kHz}$ being tested. Separately, the use of magnetoelastic resonators as planar vibrating substrates has also been evaluated for the control of cell adhesion ${ }^{48}$. A significant reduction in the adhering fibroblast cell count was found for vibration amplitudes of $150 \mathrm{~nm}$, using planar rectangular magnetoelastic resonators operating at $176 \mathrm{kHz}$.

We envision that magnetoelastic actuators can be integrated with GDDs to limit cellular adhesion on the implant surface. The actuator could be either embedded within the GDD or affixed to it prior to surgical implantation. The shear stresses generated directly by the vibration of the actuator and indirectly by the fluid agitation resulting from this vibration would reduce the adhesion of fibroblasts and endothelial cells ${ }^{45-47}$. As a result, the actuation would impede complete encapsulation by preventing cells from adhering to large portions of the implant. This is expected to increase the permeability of the surrounding tissue to the $A H$ draining from the AGDD, enabling the AGDD to better control the IOP. Post-implantation, the actuator could be deployed periodically-perhaps for only a few minutes per day-using a small external coil to generate an oscillating magnetic field. The external coil, positioned a few centimeters from the implant, would need to generate only a weak magnetic field $(<20 \mathrm{G})$ and could even be embedded in a toothbrush or a wearable device. This procedure could be safely administered by the patient or a caregiver with minimal instruction.

Although magnetoelastic materials are attractive for use in wireless sensors and actuators, the fabrication of complex 3D geometries and curved surfaces remains challenging. This is especially important for implantable applications in which such shapes are necessary to conform to biological features or devices such as the AGDD. This paper describes the design and fabrication of actuators from amorphous magnetoelastic materials and results from in vitro experiments performed with these actuators. The actuators have customized 3D shapes and curvatures to conform to an AGDD (Figure 1c). Six actuator designs are evaluated, with varying implant coverage areas and resonant frequencies. The following sections describe, in sequence, the design and modeling of the actuators, the fabrication process, and the experimental results, followed by discussion and conclusions.

\section{DESIGN AND MODELING}

\section{Design}

The complex geometry of the AGDD warrants special considerations for actuator development. The actuator should closely conform to the AGDD surface to avoid changes to the manner in which the device fits the eye and to prevent any damage to the surrounding ocular tissue. This is a design and fabrication issue because amorphous magnetoelastic materials, from which the actuators are patterned, are generally available only as flat foils. The design of the actuator must also accommodate various features on the AGDD (Figure 1c). Three perforations in the posterior portion of the implant allow the growth of fibrous tissue that "rivets" the plate body of the implant to the scleral 
surface on which it rests, whereas two are located in the anterior portion for suturing the implant in place. In addition, there are two ridges located on the posterior of the implant. The actuator design must avoid both the perforations and ridges on the plate surface.

Additionally, there are considerations related to the handling of the AGDD during the implantation procedure. During the implantation procedure, the AGDD is grasped with forceps by the surgeon (on the portion of the device posterior to the valve mechanism) and placed under the conjunctiva before it is sutured to the sclera. The actuator should avoid the area of the plate body where the forceps are typically positioned during this procedure.

To enable the design, fabrication and simulation of the complex actuator geometry, a 3D model of the AGDD was necessary. An AGDD was scanned using a Scanco $\mu C T 100$ micro-computed tomography $(\mu \mathrm{CT})$ system. A software package (Mimics ${ }^{\mathrm{TM}}$ 14, Materialise NV, Leuven, Belgium) was used to create a 3D mesh from the scan data, which could then be imported into computeraided design and finite element analysis tools. Another software module (Materialise 3-Matic ${ }^{\mathrm{TM}}$ ) was then used to repair the mesh and remove any imperfections and artifacts caused by the scanning process. The AGDD model that was created from the CT scan data is shown in Figure 1c.

Six actuator designs were evaluated, each covering different areas on the AGDD. The actuators were designed for fabrication from $29-\mu \mathrm{m}$-thick foils of an amorphous magnetoelastic alloy and, hence, are of this same thickness. Each of the actuators includes three types of elements-paddles, anchors, and springs. The Type

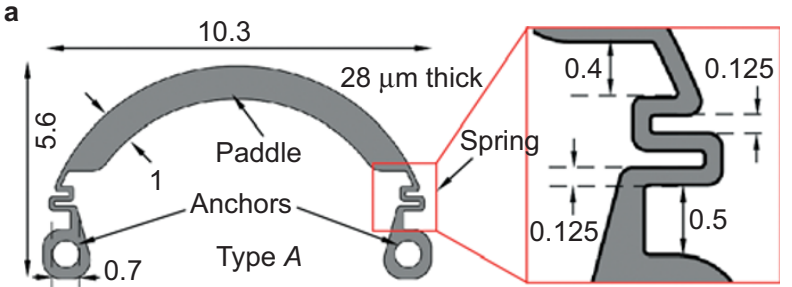

b

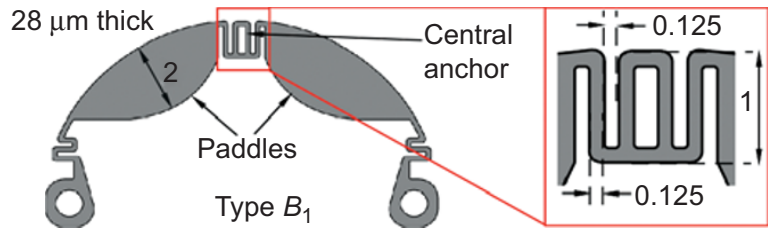

c

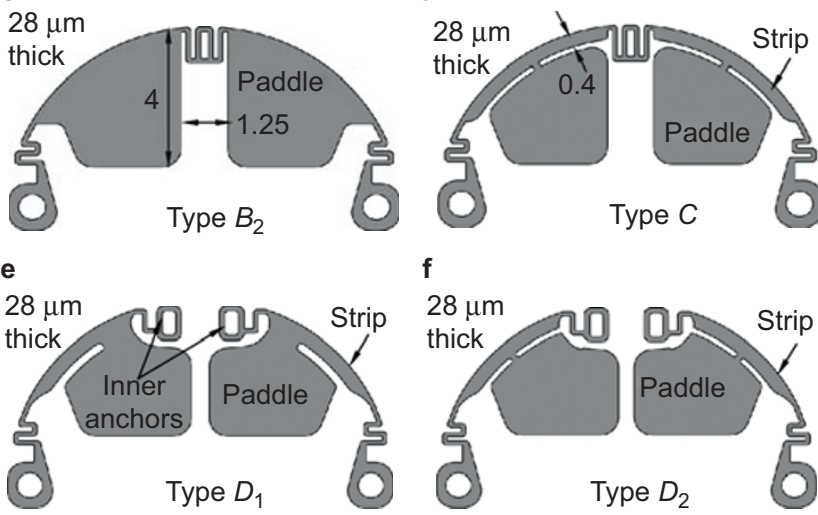

Figure 2 Dimensions of the six actuator designs (in $\mathrm{mm}$ ).
A design consists of a paddle circumscribing the posterior (downstream) portion of the plate body (Figure 2a). The resonant vibration of this paddle causes agitation and flow in the fluid in which it is immersed. The outer radius of the paddle is $5.1 \mathrm{~mm}$, and the inner radius is $4.2 \mathrm{~mm}$, making the width approximately 1 $\mathrm{mm}$. Both ends of the paddle are attached to compliant segments to relieve the stress from the anchors. The compliant segments are folded structures measuring $125 \mu \mathrm{m}$ wide, with the entire suspension on each side measuring $1.6 \times 0.75 \mathrm{~mm}^{2}$. Attached to the springs are anchors measuring $1.3 \times 1.3 \mathrm{~mm}^{2}$, each with a perforation measuring $0.7 \mathrm{~mm}$ in diameter. To anchor the actuator, an adhesive is used that, once hardened, will rivet the anchor to the plate body. The entire actuator footprint measures $10.3 \times 5.6 \mathrm{~mm}^{2}$ on a planar foil.

Type $B$ includes two similar designs with increased coverage of the plate body (Figure $2 \mathrm{~b}$ and $\mathrm{c}$ ). In addition, a central anchor is provided for added security during implantation when the AGDD is pushed underneath the conjunctiva with the forceps. The anchor measures $1.3 \times 1 \mathrm{~mm}^{2}$ and is made of beam elements that are $125 \mu \mathrm{m}$ wide. For Type $B_{1}$, the paddles measure approximately 2 $\mathrm{mm}$ wide at the widest point (Figure $2 \mathrm{~b}$ ), whereas for Type $B_{2}$, they measure $4 \mathrm{~mm}$ at the widest point (Figure $2 \mathrm{c}$ ). The outer springs and anchors are identical to those of the Type $A$ actuator. Both designs measure approximately $10.3 \times 5.6 \mathrm{~mm}^{2}$.

Type $C$ is a hybrid of Types $A$ and $B$, with wide paddles attached to a thin strip circumscribing a portion of the posterior of the plate body, divided by the central anchor (Figure $2 \mathrm{~d}$ ). The central anchor is identical to that in the Type $B$ actuators. The outer radius of the strip is $5.1 \mathrm{~mm}$, and the inner radius is $4.7 \mathrm{~mm}$, making the strip approximately $0.4 \mathrm{~mm}$ wide. The paddles attached to the spring measure $3.25 \mathrm{~mm}$ at the widest point. The outer anchors and springs are identical to those of the previous actuators. The entire actuator measures $10.3 \times 5.6 \mathrm{~mm}^{2}$.

Finally, Type $D$ includes two designs with physically separate paddles on each half of the AGDD (Figure 2e and f). Each paddle is attached to a strip that circumscribes a portion of the plate body, with one end of the strip attached to the outer springs and anchor and the other end attached to the inner spring and anchor. Types $D_{1}$ and $D_{2}$ differ on the point of attachment to the strip. In Type $D_{1}$, the attachment is located toward the end of the strip: a flexural motion of the strip results in a torsional motion of the paddle (Figure 3a). Type $D_{2}$ has a central attachment: a flexural motion of the strip results in a transverse motion of the paddles (Figure $3 \mathrm{~b}$ ). For both designs, the outer radius of the strip is $5.1 \mathrm{~mm}$ and the inner radius is 4.7 $\mathrm{mm}$, making the strip approximately $0.4 \mathrm{~mm}$ wide. The inner anchors measure $1 \times 0.7 \mathrm{~mm}^{2}$ for each of the paddles in both

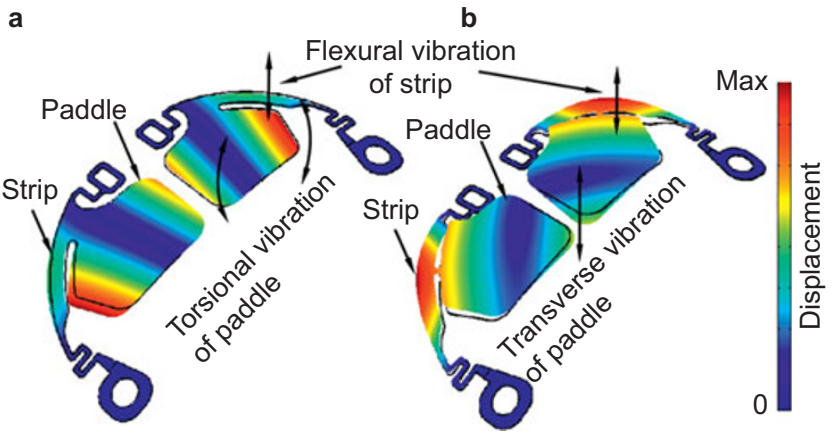

Figure 3 Target resonant modes for Type $D$ actuators: (a) Type $D_{1}$ and (b) Type $D_{2}$. The different points of attachment of the paddles to the strip result in different paddle vibration modes. 
designs. The inner springs measure $0.7 \times 0.6 \mathrm{~mm}^{2}$ and are made from beam elements that are $125 \mu \mathrm{m}$ wide. The outer anchors and springs for each of the paddles are identical to those of the previous actuators. The entire actuator measures $10.3 \times 5.6 \mathrm{~mm}^{2}$.

The overall dimensions of the actuators are designed to fit the Model FP7 AGDD. A meandering shape for the spring provides compliance within a small form factor. The width of the folded structures that make up the compliant spring elements (i.e., 125 $\mu \mathrm{m})$ represents the lower limit of the photochemical machining (PCM) fabrication process used, further details of which are provided in the "Fabrication" Section. The anchors are designed to be sufficiently large to allow easy manual attachment. The variations in the features of the different actuator types are designed to provide empirical information on the value of these various features. Type $B$ designs have a larger area over the valve plate body than the Type $A$ design to determine the impact of this area on the flow. Type $C$ actuator paddles are attached to the strip to determine the impact on paddle compliance and the vibration displacement amplitude. Type $D$ designs have physically separate paddles on each half of the AGDD to accommodate any deformation of the soft AGDD plate body.

The flexibility of the AGDD plate body is an important feature of the AGDD that enhances its performance ${ }^{49}$. The compliant spring elements of the actuator, the overall thin structure, and the fact that there are only two or three anchor points ensure that the flexibility of the AGDD plate body is not compromised. Through finite element simulations in COMSOL Multiphysics 4.4 (COMSOL AB, Stockholm, Sweden), the out-of-plane compliance of the Type A actuator was estimated to be nearly six orders of magnitude higher than that of the plate body. Finally, by locating the actuator sufficiently far from the valve mechanism, potential interference with this mechanism is avoided.

\section{Modeling}

Magnetoelastic materials exhibit an elastic response to applied magnetic fields in an effect termed Joule magnetostriction. As a result, magnetoelastic structures can be excited to mechanical resonance using oscillating magnetic fields. An inverse effect, called the Villari effect, describes the magnetization resulting from applied mechanical strain. In a vibrating structure, this occurs concurrently with Joule magnetostriction. The coupling between the magnetization and the mechanical stress or strain can be expressed by a pair of equations. For a one-dimensional system $^{50}$, these are as follows:

$$
\begin{aligned}
& \varepsilon=\left.\frac{\partial \varepsilon}{\partial \sigma}\right|_{H} \sigma+\left.\frac{\partial \varepsilon}{\partial H}\right|_{\sigma} H \\
& B=\left.\frac{\partial B}{\partial \sigma}\right|_{H} \sigma+\left.\frac{\partial B}{\partial H}\right|_{\sigma} H
\end{aligned}
$$

where $\sigma$ is stress, $\varepsilon$ is strain, $B$ is magnetic flux density, and $H$ is magnetic field intensity. The partial derivatives are as follows: $\left.\frac{\partial \varepsilon}{\partial \sigma}\right|_{H}$ is the compliance at constant $H, S^{H} ;\left.\frac{\partial B}{\partial H}\right|_{\sigma}$ is the permeability at constant stress, $\mu^{\sigma}$; and $\left.\frac{\partial \varepsilon}{\partial H}\right|_{\sigma}$ and $\left.\frac{\partial B}{\partial \sigma}\right|_{H}$ are both represented by $d$, the magnetostrictive coefficient. The strength of vibration is determined by the magnetostrictive coefficient, $d$, which is dependent on the direct current (DC) bias conditions. For maximum resonant response, the $D C$ magnetic field should bias the material where $d$ is high.

The resonant behavior of the actuators was simulated in COMSOL Multiphysics 4.4. Coupled solid mechanics and magnetic field physics (approximated by Equations (1) and (2)) were used to simulate the behavior of the magnetoelastic material in an oscillating magnetic field. The mechanical and magnetic properties used in this simulation were derived from manufacturer data sheets and previous reports ${ }^{51-53}$. The damping due to the liquid environment was obtained experimentally from laser displacement meter measurements performed on the final fabricated actuators. The details of this method are provided in "EXPERIMENTAL METHODS AND RESULTS" section.

The resonant frequencies and mode shapes exhibiting the maximum vibration amplitudes are shown in Figure 4. The simulations predicted multiple flexural modes for Type $A$ actuators, with the third mode (at $900 \mathrm{~Hz}$ ) exhibiting the maximum resonant amplitudes. For Type $B$, the resonant frequencies with the largest displacements were $4 \mathrm{kHz}$ and $3.5 \mathrm{kHz}$ for Types $B_{1}$ and $B_{2}$, respectively. The Type $C$ actuator had a low resonant frequency of $450 \mathrm{~Hz}$ that resulted in large displacements. For Type $D$ actuators, target resonant modes were identified at 560 $\mathrm{Hz}$ and $850 \mathrm{~Hz}$ for Types $D_{1}$ and $D_{2}$, respectively.

\section{FABRICATION}

Magnetoelastic materials are available in both crystalline and amorphous forms. Of particular interest for microscale sensing and actuation applications are amorphous magnetoelastic alloys that are commercially available as planar foils. Microfabrication techniques such as micro-electrodischarge machining $(\mu \mathrm{EDM})^{54}$ and $\mathrm{PCM}^{55}$ can be used to pattern these materials with spatial resolutions down to a few tens of micrometers.

In contrast to crystalline materials, amorphous magnetoelastic alloys operate at a lower bias field, thus reducing the size of the biasing magnet or electric current that is necessary ${ }^{14,56}$. Moreover, amorphous magnetoelastic materials can be tailored to have a high magnetomechanical coupling coefficient (i.e., the conversion efficiency between magnetic and mechanical energy) through annealing in the presence of a magnetic field; this results in a stronger resonant response to a given stimulus ${ }^{57,58}$. With
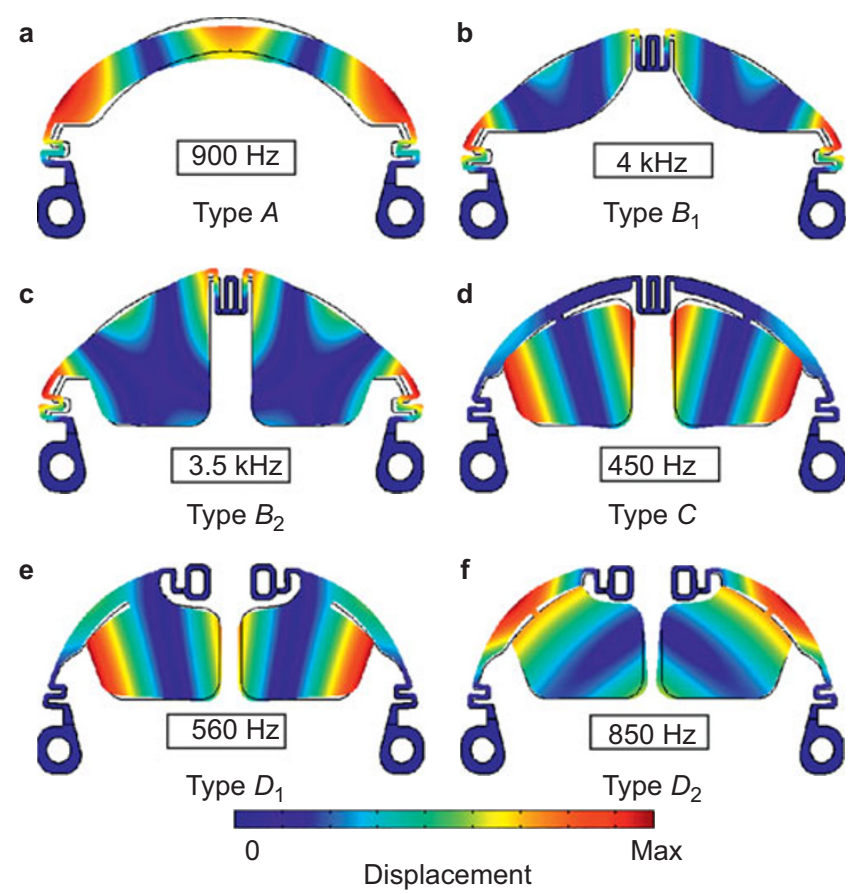

Figure 4 Simulated resonant frequencies and corresponding mode shapes showing maximum vibration amplitudes. 
these considerations in mind, amorphous alloys were selected for use in this work.

For this work, the actuators were fabricated from $29-\mu \mathrm{m}$-thick foils of Metglas $2826 \mathrm{MB}\left(\mathrm{Fe}_{40} \mathrm{Ni}_{38} \mathrm{Mo}_{4} \mathrm{~B}_{18}\right)$ (Metglas, Inc., Conway, SC, USA). This material has a saturation magnetostriction of 12 ppm and a DC permeability greater than $50000^{51}$. The high permeability is beneficial for attracting and directing the biasing magnetic field along the actuator, allowing some leeway in the alignment of the DC magnetic field coils.

The fabrication of the actuators is a two-step process: patterning of the thin-film magnetoelastic alloy, followed by thermal annealing to induce the desired curvature. The actuators are patterned from flat sheets of Metglas 2826 MB using PCM (Kemac Technology, Inc., Azusa, CA, USA). In this process, the foil is first coated with a photoresist, which is lithographically patterned by ultraviolet exposure. The exposed regions of the substrate (not covered by the mask) are then etched away using a spray of etchant. Advantages of this technique include burr-free fabrication and retention of the magnetic properties of the material. The process flow for PCM is shown in Figure $5 a-d$.

To obtain an actuator with the necessary curvature, a patterned sheet of Metglas 2826 MB must be annealed in a mold at elevated temperature to remove the stress induced by the applied curvature. A longer annealing duration or higher temperature causes the initially flat structure to more closely conform to the curvature of the annealing mold. The disadvantage is that a higher temperature also results in embrittlement. Furthermore, a temperature that is too high can also lead to crystallization of the material or loss of ferromagnetism (if it exceeds the Curie point), resulting in a reduction in permeability ${ }^{59}$. The Curie temperature for Metglas $2826 \mathrm{MB}$ is $353{ }^{\circ} \mathrm{C}$, and its temperature of crystallization is $440{ }^{\circ} \mathrm{C}^{60,61}$. Conversely, a shorter annealing duration or lower temperature prevents the shape of the mold from being completely transferred to the final actuator. Preliminary experiments focused on inducing curvature in the actuators a

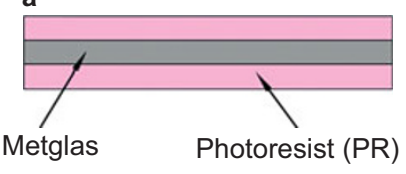

Laminate PR

b

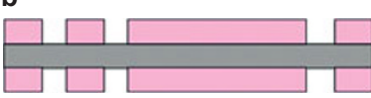

Expose and develop PR

c

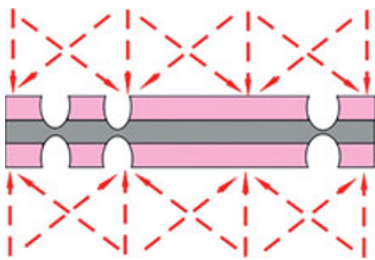

Spray etchant

d

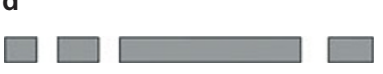

Strip PR

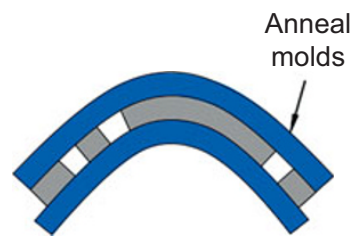

Anneal in mold

f

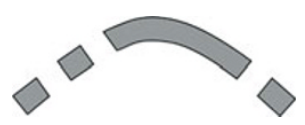

Remove from mold

g

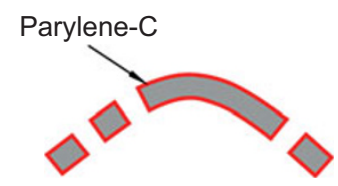

Deposit parylene-C
Figure 5 Process flow for actuator fabrication. Steps (a)-(d) result in the patterning of the magnetoelastic foil. Steps $(\mathbf{e})-(\mathbf{g})$ impart the required curvature and biocompatibility. indicated that the recoil is $25-40 \%$ of the target deformation upon removal from the mold. The shape of the mold must account for this partial recoil. For this study, it was found that annealing at $275^{\circ} \mathrm{C}$ for 12 hours in a mold with an exaggerated curvature yielded a desirable outcome. It is important that the curvature of the actuator conform to the AGDD. Inexact curvature can result in damage to the surrounding tissue, difficulty in surgical implantation of the device, and reduced actuation efficacy due to the larger separation from the implant surface.

The annealing mold consisted of two plates (with exaggerated curvatures) between which a PCM-patterned sheet of Metglas $2826 \mathrm{MB}$ was sandwiched during the annealing process (Figure 5e). The annealed actuators were then coated with a 1- $\mu \mathrm{m}$-thick layer of Parylene- $C$ to prevent corrosion in the aqueous testing environment and to provide in vivo biocompatibility ${ }^{62}$ (Figure $5 \mathrm{~g}$ ). The as-fabricated actuators (prior to annealing) and the annealed actuators are shown in Figure 6.

The plates used for annealing were designed based on the 3Dscanned model of the AGDD and fabricated by direct metal laser sintering (DMLS) of a cobalt-chrome alloy (GPI Prototype and Manufacturing Services, Lake Bluff, IL, USA). In the DMLS process, which is an additive manufacturing process, a laser is used to sinter powdered metal layer by layer to create the required solid structure ${ }^{63}$. In addition to the plates used for annealing, DMLS was also used to fabricate test plates that mimicked the curvature of the AGDD. During the experimental measurements, the actuators were attached to

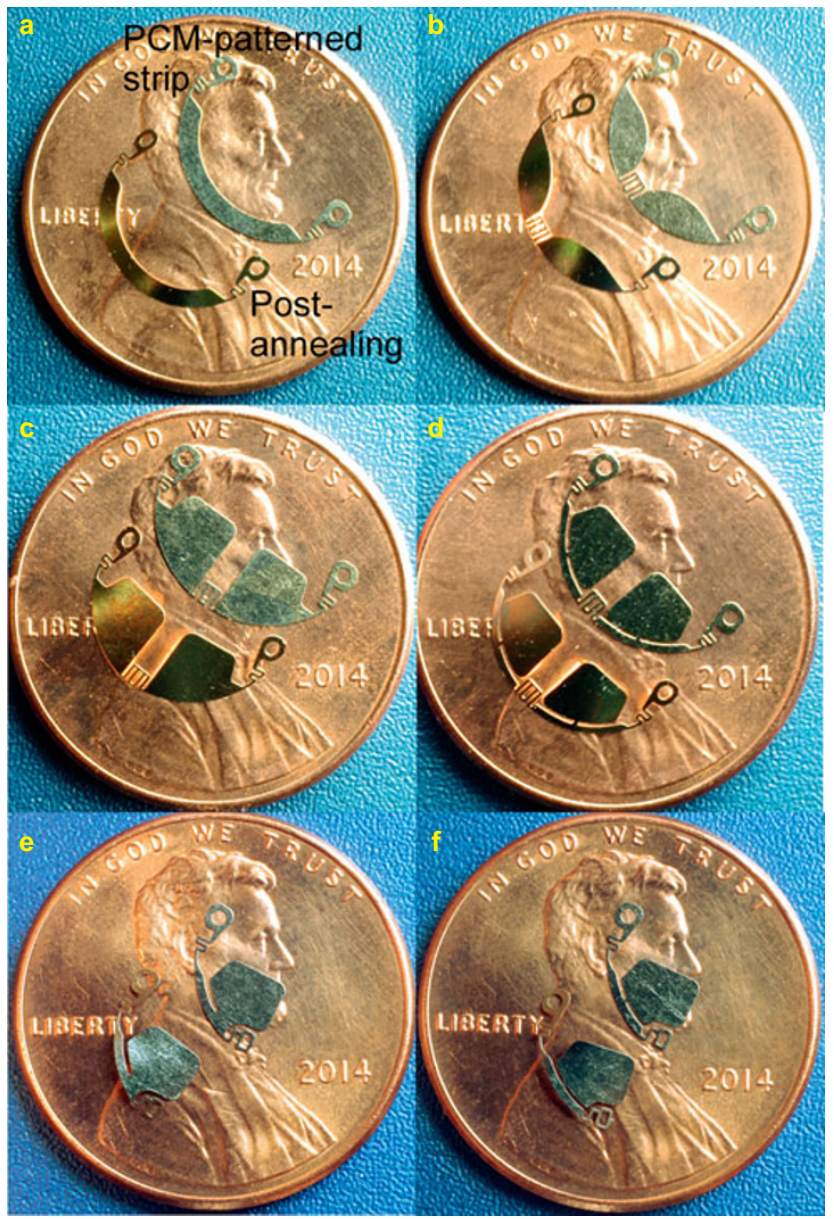

Figure 6 Fabricated actuators after photochemical machining and annealing: (a) Type $A_{1}$ (b) Type $B_{1}$, (c) Type $B_{2}$, (d) Type $C_{1}$ (e) Type $D_{1}$, and (f) Type $D_{2}$. 


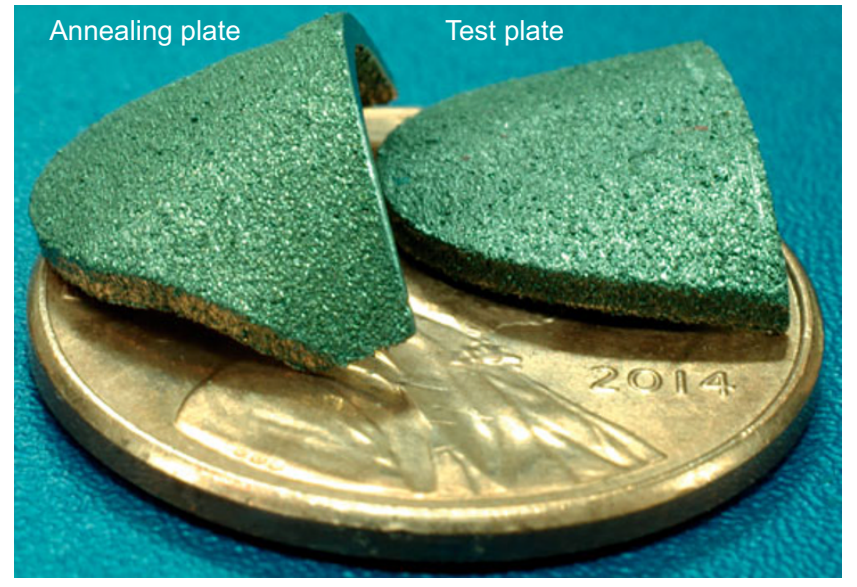

Figure 7 Fabricated plates for annealing and actuator testing. Each patterned foil of Metglas $2826 \mathrm{MB}$ was sandwiched between two annealing plates during the annealing process. The test plate mimicked the curvature of the AGDD.

these test plates to replicate the curvature of the AGDD. The fabricated plates used for annealing and testing are shown in Figure 7.

\section{EXPERIMENTAL METHODS AND RESULTS}

The annealed and coated actuators were experimentally evaluated using an optical approach to determine the resonant modes that generated the maximum liquid motion. Each actuator was placed in an aqueous suspension of 3.1- $\mu \mathrm{m}$-diameter particles (Fluoro-Max dyed green aqueous fluorescent particles, Thermo Fisher Scientific Inc., Waltham, MA, USA) at a concentration of 6 $\mathrm{ng} \mathrm{ml}^{-1}$. These particles have an excitation wavelength of $468 \mathrm{~nm}$ and an emission wavelength of $508 \mathrm{~nm}$. The motion of the particles was observed under a microscope (Olympus SZX12 stereo microscope, Olympus Corporation, Tokyo, Japan), and the images were recorded using a digital camera (Pentax K7, Ricoh Company, Ltd., Tokyo, Japan). The particle trajectories were captured in long-exposure ( $0.6 \mathrm{~s})$ photographs acquired through the microscope. The particle velocities were estimated based on the distances traveled by the particles during the exposure time of the photographs. All measurements were collected at $23{ }^{\circ} \mathrm{C}$.

Magnetic biasing was provided by a set of Helmholtz coils that generated a DC magnetic field, and activation was achieved using a second set of coils, coaxial to the first, that transmitted an alternating current (AC) magnetic field (Figure 8). The DC biasing field measured $20 \mathrm{G}$, whereas the $A C$ excitation magnetic field measured $4 \mathrm{G} \mathrm{rms}$. The frequency of the AC excitation magnetic field varied from $0.1 \mathrm{kHz}$ to 5 $\mathrm{kHz}$. Each annealed actuator was attached to the DMLS-fabricated test plate at its anchor points using cyanoacrylate adhesive. The coils generating the AC excitation magnetic field were located $25-30 \mathrm{~mm}$ away from the anchored actuator.

Figure 9 shows streaks of particle motion generated by a Type $A$ actuator, as observed in a long-exposure photograph. At this location, the particles traveled an average distance of $149 \mu \mathrm{m}$ in $0.6 \mathrm{~s}$. The velocity of the particles was thus estimated to be $248 \mu \mathrm{m} \mathrm{s}^{-1}$. Figure 10 shows the particle velocities estimated for each of the actuators using this technique. The regions of the actuators exhibiting particle motion correspond to the antinodes of that particular resonant mode. The mode shapes indicated by the observed antinodes correspond to the simulated mode shapes shown in Figure 3 . The Type $A$ actuator had six regions where particle flow was generated, with an average flow velocity of 230 $\mu \mathrm{m} \mathrm{s}^{-1}$ at a resonant frequency of $890 \mathrm{~Hz}$. The flow pattern indicated the third flexural mode of the paddle, confirming the

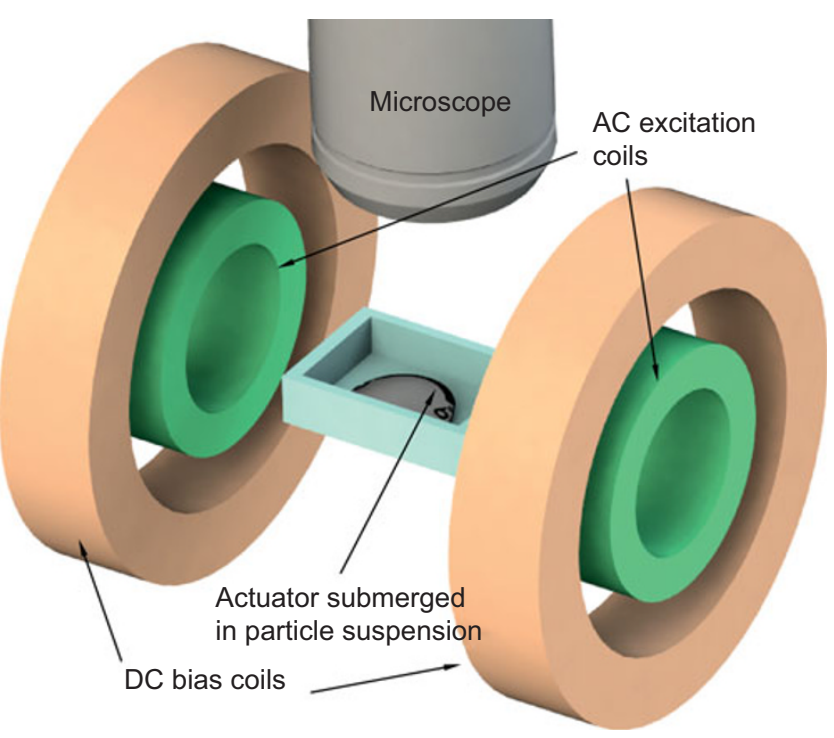

Figure 8 Test setup for the particle flow measurements. DC bias coils generated the DC magnetic field for biasing the actuator in the high-magnetostrictivity region. AC excitation coils generated the $A C$ magnetic field to induce actuator vibration.

simulated mode shape. Both Type $B$ actuators had five regions where particle flow was generated on each paddle. For Type $B_{1}$, an average flow velocity of $200 \mu \mathrm{m} \mathrm{s}^{-1}$ was recorded, whereas for Type $B_{2}$, an average flow velocity of $135 \mu \mathrm{m} \mathrm{s}^{-1}$ was found. The resonant frequency was $3.8 \mathrm{kHz}$ for Type $B_{1}$ and $4.7 \mathrm{kHz}$ for Type $B_{2}$. Type $C$ actuator had five regions on each paddle where particle flow was generated, with an average flow velocity of $118 \mu \mathrm{m} \mathrm{s}^{-1}$ at a resonant frequency of $520 \mathrm{~Hz}$. The Type $D_{1}$ actuator had two regions on each paddle where particle flow was generated, with an average flow velocity of $73 \mu \mathrm{m} \mathrm{s}^{-1}$ at a resonant frequency of 740 Hz. Finally, the Type $D_{2}$ actuator had four regions on each paddle where particle flow was generated, with an average flow velocity of $70 \mu \mathrm{m} \mathrm{s}^{-1}$ at a resonant frequency of $740 \mathrm{~Hz}$.

To estimate the influence of the viscous damping caused by the aqueous environment surrounding the plate body of the AGDD on

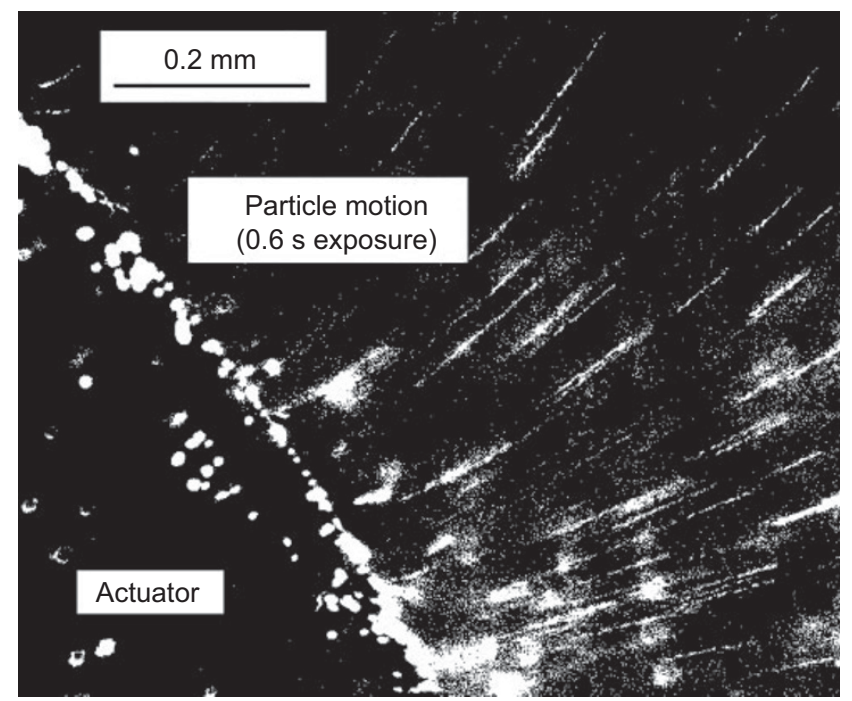

Figure 9 Moving fluorescent microparticles observed as streaks in a photograph of a Type $A$ actuator resonating at $890 \mathrm{~Hz}$ with $0.6 \mathrm{~s}$ of exposure. Image thresholding was applied to improve visibility. 
the vibration amplitudes and the quality factor of the resonance, the mechanical frequency response was measured for the Type $A$ actuator. A laser displacement sensor (Model LK-G32, Keyence Corporation, Osaka, Japan) was used to measure the vibration amplitudes. The displacement of the center of the Type $A$ actuator (in air) as a function of frequency for an $A C$ excitation magnetic field of amplitude $0.2 \mathrm{G}$ is shown in Figure 11a. The experiment was repeated with the actuator submerged in water (at a depth of less than $1 \mathrm{~mm}$ ) (Figure 11b). The excitation field was increased to $2 \mathrm{G}$ amplitude to generate a measureable response in this highdamping environment. Peak amplitudes of $2.4 \mu \mathrm{m}$ were observed in air, whereas in water (with a 10-fold increase in the applied magnetic field), the peak amplitude was approximately $1.5 \mu \mathrm{m}$.

\section{DISCUSSION}

The flexural modes of vibration of the actuators are difficult to detect using magnetic means because the vibrations cause simultaneous tensile and compressive strains. These strains have opposing effects on the generated magnetic field, making detection of resonant peaks using inductive coupling methods difficult. The expected functionality of the actuator is that of prevention of cell adhesion and removal of adhered cells through vibration. The direct observation of these effects of vibration, through particle flow measurements, offers a superior assessment of this functionality. This approach also enables the localization of regions of high vibration amplitudes, which would not be possible using an inductive coupling method.

The particle velocities, on average, were inversely proportional to the area of the actuator. This was anticipated, as a wider paddle transfers more energy to the surrounding liquid. However, a wider paddle also covers a greater portion of the AGDD and is subject to more damping. In future designs, the paddles may be perforated. a

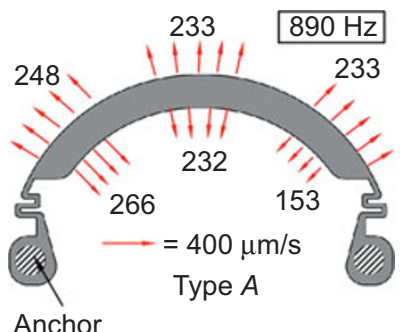

c

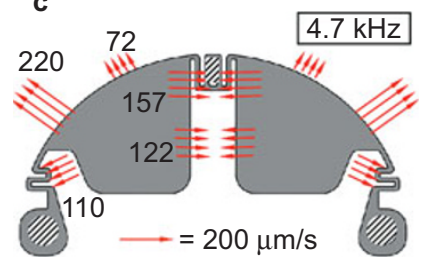

Type $B_{2}$

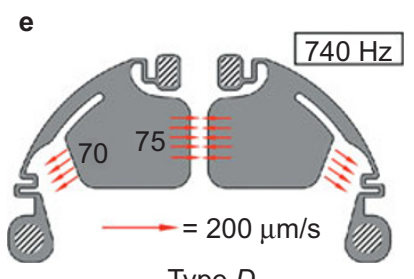

Type $D_{1}$ b

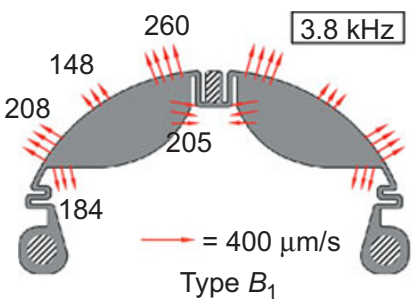

d

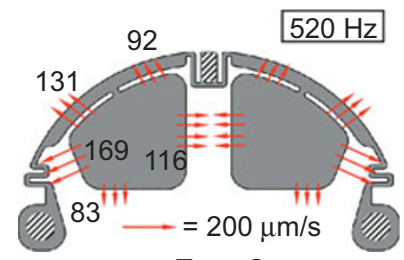

Type $C$

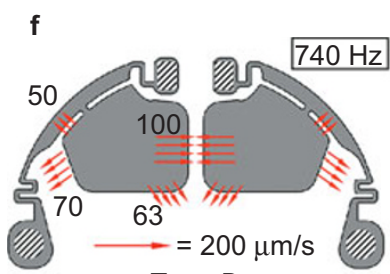

Type $D_{2}$
Figure 10 Measured resonant frequencies and particle velocities (in $\mu \mathrm{m} \mathrm{s}^{-1}$ ) for the actuators in water.
A possible concern associated with the use of amorphous magnetic alloys such as Metglas $2826 \mathrm{MB}$ for implantable devices is compatibility with magnetic resonance imaging (MRI). The small form factor of the actuators and the low-eddy current and hysteresis losses ${ }^{64}$ of amorphous magnetic alloys diminish tissue heating. Preliminary calculations show that for a $64-\mathrm{MHz}$ system with a $10-\mu$ T oscillating field magnitude (i.e., a $B_{1}$ field), the tissue heating due to eddy current and hysteresis losses is within the specifications of the IEC 60601-2-33 standard, which establishes the safety limits in an MRI environment ${ }^{65,66}$. The small form factor of the actuators also ensures that any artifacts in the MRI image (due to the high permeability of the material) are localized to the immediate vicinity of the actuators.

Despite the fact that the target application of the actuators described in this work is to minimize cell adhesion through vibration, it is quite possible that some accumulation of cells on the actuator surfaces may occur nonetheless. Any change in the resonant frequency due to such tissue loading can be accommodated by using a wideband AC excitation magnetic field rather than a single tone. In addition, previous studies have reported appreciable resonant amplitudes for significant mass loading (in excess of five times the resonator mass) ${ }^{10}$. Finally, Parylene-C, with which the actuators are coated, exhibits excellent long-term stability and efficacy as a biocompatible material ${ }^{62}$. It does not exhibit any swelling in aqueous environments ${ }^{67}$. As a result, Parylene-C-coated magnetoelastic actuators are expected to remain stable in vivo.

The simulation model, in conjunction with frequency response measurements, enabled the determination of the energy transfer efficiency between the applied magnetic energy and the induced kinetic energy of the liquid. The quality factors of the Type $A$ actuator in air and water were determined from the frequency response plots shown in Figure 10 . The quality factor $Q$ is approximately equal to $f_{0} / \Delta f$, where $f_{0}$ is the resonant frequency and $\Delta f$ is the $3-\mathrm{dB}$ bandwidth. From Figure $11 \mathrm{a}$, the quality factor in air is given by:

$$
Q_{\text {air }} \approx \frac{f_{0}}{\Delta f}=\frac{2060}{15}=137
$$

Similarly, from Figure 10b, the quality factor in water is given by:

$$
Q_{\text {water }} \approx \frac{f_{0}}{\Delta f}=\frac{900}{75}=12
$$

The quality factor in water, $Q_{\text {water, }}$ can be expressed as the following equation of its constituent parts:

$$
\frac{1}{Q_{\text {water }}}=\frac{1}{Q_{\text {int }}}+\frac{1}{Q_{\text {visc }}}
$$

where $Q_{\text {int }}$ is the intrinsic loss, including material losses and anchor loss, and $Q_{\text {visc }}$ is the loss due to viscous damping in water. lgnoring viscous losses in air (under the assumption that intrinsic losses dominate in air), $Q_{\text {air }}$ is approximately equal to $Q_{\text {int }}$. Hence, from Equation (5), $Q_{\text {visc }}$ is approximately equal to 13 .

The experimentally determined quality factors and frequency response plots were used to model the damping in the customcoupled magnetomechanical simulation implemented in COMSOL Multiphysics. The frequency response as simulated by COMSOL is shown in Figure 10b, superimposed on the measured response. The simulated average magnetic energy, $E_{\mathrm{mag}}$ delivered to the resonator by the excitation coils is $2.9 \times 10^{-10} \mathrm{~J}$. The simulated mechanical energy, $E_{\text {mech, }}$ of the resonator is $3 \times 10^{-12} \mathrm{~J}$. The energy transferred per cycle (through viscous damping) to the liquid, $E_{\text {liquid, }}$ is $E_{\text {liquid }} / Q_{\text {visc }}$ and is equal to $2.3 \times 10^{-13} \mathrm{~J}$. Finally, 

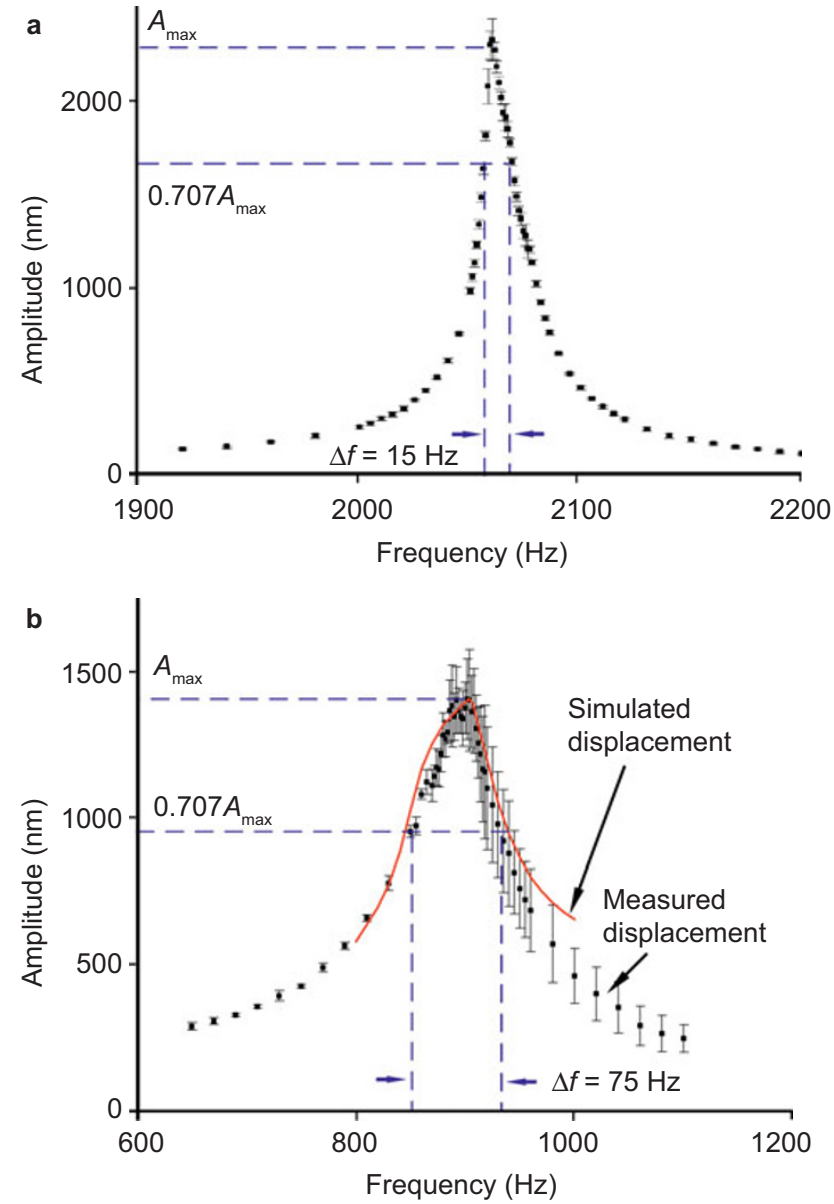

Figure 11 Frequency responses measured using a laser displacement meter for the Type $A$ actuator in (a) air and (b) water. The plotted amplitudes were measured at the center of the actuator paddle.

the conversion efficiency for the energy transferred from the input magnetic field to the liquid is estimated to be $E_{\text {liquid }} / E_{\text {mag, }}$ i.e., $0.08 \%$.

The magnetomechanical coupling coefficient, $k^{2}$, that determines the energy conversion efficiency between the mechanical and magnetic domains is given by an expression that depends solely on the material parameters ${ }^{68}$ :

$$
k^{2}=\frac{d^{2}}{s^{H} \mu^{\sigma}}
$$

where $d$ is the small signal magnetostrictivity, $S^{H}$ is the stiffness at constant magnetic field, and $\mu^{\sigma}$ is the permeability at constant stress. As stated in "Fabrication" section, in an amorphous alloy, this property can be tuned by annealing in a magnetic field. This provides an avenue for improving the efficiency of the energy transfer into the liquid.

The results for the Type $A$ actuator in water indicate vibration amplitudes exceeding $1 \mu \mathrm{m}$. Extrapolating these results to the other designs using the particle velocity measurements, it is safe to assume vibration amplitudes on the order of hundreds of nanometers for all fabricated actuators. Vibration amplitudes and frequencies on the order reported here have previously been shown to reduce cellular adhesion ${ }^{46,48}$. Hence, it can be concluded that the actuators fabricated in this study show vibration amplitudes that are sufficient to impact cell adhesion and proliferation, and can ultimately result in improving the effectiveness of the AGDD in lowering the IOP and preventing vision loss from glaucoma.

Whereas the experimental results reported here for the wireless actuators demonstrate the ability to generate liquid flow, further studies are needed to establish the appropriate usage modality for fibrosis mitigation. Parameters such as amplitude, duration, and periodicity could influence the efficacy of the technique and can only be determined through in vivo studies in animal models.

\section{CONCLUSION}

This paper describes the design and fabrication of passive, wireless magnetoelastic resonant actuators for microfluidic actuation targeted at mitigating fibrosis in a glaucoma drainage device, as well as the results of in vitro experiments with these actuators. By means of three-dimensional scanning, printing, and molding techniques, complex geometries were fabricated from commercially available amorphous magnetoelastic foils. Particle flow and laser displacement meter measurements were performed to characterize the operation of the actuators in an aqueous environment. Type $A$ and $B$ actuators exhibited the highest particle flow velocities among the set that was evaluated. Type $B$ actuators, with a central anchor for improved surgical compatibility, showed promise as a viable and practical enhancement for the AGDD. Future efforts should be directed at establishing the most suitable usage modality for effective mitigation of fibrosis. This concept can also be extended to other biomedical implants in which microfluidic actuation is required.

\section{ACKNOWLEDGEMENTS}

The authors acknowledge Metglas, Inc., for providing foil samples for use in this project. The authors also thank Dr. Scott Green for useful discussions, Ms. Yushu Ma and Dr. Yutao Qin for their assistance with the Parylene-C deposition, and Ms. Rose Anderson for her assistance with the graphics. This effort was supported by a grant from the University of Michigan.

\section{COMPETING INTERESTS}

The authors declare no conflict of interest.

\section{REFERENCES}

1 Liu NC, Lian MR, Cantey J. Transverse magnetic field annealed amorphous magnetomechanical elements for use in electronic article surveillance system and method of making same. United States Patent 5469140, Nov. 21, 1995.

2 Grimes CA, Roy SC, Rani S et al. Theory, instrumentation and applications of magnetoelastic resonance sensors: A review. Sensors 2011; 11: 2809-2844.

3 Traxler S, Kosel J, Pfützner $\mathrm{H}$ et al. Contactless flow detection with magnetostrictive bilayers. Sensors and Actuators A: Physical 2008; 142: 491-495.

4 Grimes CA. Simultaneous measurement of liquid density and viscosity using remote query magnetoelastic sensors. Review of Scientific Instruments 2000; 71: 3822-3824.

5 Calkins FT, Flatau AB, Dapino MJ. Overview of magnetostrictive sensor technology. Journal of Intelligent Material Systems and Structures 2007; 18: 1057-1066.

6 Karl WJ, Powell AL, Watts $\mathrm{R}$ et al. A micromachined magnetostrictive pressure sensor using magneto-optical interrogation. Sensors and Actuators A: Physical 2000; 81: 137-141.

7 Pepakayala V, Green SR, Gianchandani YB. Passive wireless strain sensors using microfabricated magnetoelastic beam elements. Journal of Microelectromechanical Systems 2014; 23: 1374-1382.

8 Hall DL, Flatau AB. Nonlinearities, harmonics, and trends in dynamic applications of Terfenol-D. Smart Structures and Materials 1993; 1917: 929-939.

9 Tang J, Green SR, Gianchandani YB. Miniature wireless magnetoelastic resonant motor with frequency selectable bidirectional rotation. Journal of Microelectromechanical Systems 2013; 22: 730-738.

10 Green SR, Gianchandani YB. Wireless magnetoelastic monitoring of biliary stents. Journal of Microelectromechanical Systems 2009; 18: 64-78.

11 Viswanath A, Green SR, Kosel J et al. Metglas-Elgiloy bi-layer, stent cell resonators for wireless monitoring of viscosity and mass loading. Journal of Micromechanics and Microengineering 2013; 23: 025010.

12 Tang J, Green SR, Gianchandani YB. Scalable, high-performance magnetoelastic tags using frame-suspended hexagonal resonators. Journal of Micromechanics and Microengineering 2014; 24: 065006.

13 Shen W, Zhang Z, Horikawa S et al. Time domain characterization of magnetoelastic sensors: A pulse method for resonance frequency determination. Reviews of Scientific Instruments 2010; 81: 084702. 
14 Green SR, Gianchandani YB. Tailored magnetoelastic sensor geometry for advanced functionality in wireless biliary stent monitoring systems. Journal of Micromechanics and Microengineering 2010; 20: 075040.

15 Kouzoudis D, Grimes $C$. The frequency response of magnetoelastic sensors to stress and atmospheric pressure. Smart Materials and Structures 2000; 9: 885-889.

16 Quigley HA, Broman AT. The number of people with glaucoma worldwide in 2010 and 2020. British Journal of Ophthalmology 2006; 90: 262-267.

17 Resnikoff S, Pascolini D, Etya'ale D et al. Global data on visual impairment in the year 2002. Bulletin of the World Health Organization 2004; 82: 844-851.

18 Migdal C, Hitchings R. Control of chronic simple glaucoma with primary medical, surgical and laser treatment. Transactions of the Ophthalmological Societies of the United Kingdom 1986; 105: 653-656.

19 Toris CB. Pharmacotherapies for Glaucoma. Current Molecular Medicine 2010; 10: 824-840.

20 Thomas JV, Simmons RJ, Belcher CD. Argon laser trabeculoplasty in the presurgical glaucoma patient. Ophthalmology 1982; 89: 187-197.

21 Cairns JE. Trabeculectomy. Preliminary report of a new method. American Journal of Ophthalmology 1968; 66: 673-679.

22 Gedde SJ, Schiffman JC, Feuer WJ et al. Treatment outcomes in the tube versus trabeculectomy study after one year of follow-up. American Journal of Ophthalmology 2007; 143: 9-22.

23 Loeffler KU, Jay JL. Tissue response to aqueous drainage in a functioning Molteno implant. British Journal of Ophthalmology 1988; 72: 29-35.

24 Molteno ACB, Fucik M, Dempster AG et al. Otago glaucoma surgery outcome study: Factors controlling capsule fibrosis around Molteno implants with histopathological correlation. Ophthalmology 2003; 110: 2198-2206.

25 Eibschitz-Tsimhoni M, Schertzer RM, Musch DC et al. Incidence and management of encapsulated cysts following Ahmed glaucoma valve insertion. Journal of Glaucoma 2005; 14: 276-279.

26 Wilcox M, Kadri OA. Force and geometry determine structure and function of glaucoma filtration capsules. Ophthalmologica 2007; 221: 238-243.

27 Lee JW, Park WY, Kim EA et al. Tissue response to implanted Ahmed glaucoma valve with adjunctive amniotic membrane in rabbit eyes. Ophthalmic Research 2014; 51: 129-139.

28 Philipp W, Klima G, Miller K. Clinicopathological findings 11 months after implantation of a functioning aqueous-drainage silicone implant. Graefe's Archive for Clinical and Experimental Ophthalmology 1990; 228: 481-486.

29 Cameron JD, White TC. Clinico-histopathological correlation of a successful glaucoma shunt implant. Ophthalmology 1988; 95: 1189-1194.

30 Hong CH, Arosemena A, Zurakowski D et al. Glaucoma drainage devices: A systematic literature review and current controversies. Survey of Ophthalmology 2005; 50: 48-60.

31 Choritz L, Koynov K, Renieri G et al. Surface topographies of glaucoma drainage devices and their influence on human tenon fibroblast adhesion. Investigative Ophthalmology and Visual Science 2010; 51: 4047-4053.

32 Ayyala RS, Michelini-Norris B, Flores A et al. Comparison of different biomaterials for glaucoma drainage devices: Part 2. Archives of Ophthalmology 2000; 118 1081-1084.

33 Jensen C, Gurevich L, Patriciu A et al. Increased connective tissue attachment to silicone implants by a water vapor plasma treatment. Journal of Biomedical Material Research Part A 2012; 100: 3400-3407.

34 Zhang BGX, Myers DE, Wallace GG et al. Bioactive coatings for orthopaedic implants-recent trends in development of implant coatings. International Journal of Molecular Sciences 2014; 15: 11878-11921.

35 Chu P. Plasma-surface modification of biomaterials. Materials Science and Engineering: R: Reports 2002; 36: 143-206.

36 Ross EA, Batich CD, Clapp WL et al. Tissue adhesion to bioactive glass-coated silicone tubing in a rat model of peritoneal dialysis catheters and catheter tunnels. Kidney International 2003; 63: 702-708.

37 Den Braber ET, De Ruijter JE, Jansen JA. The effect of a subcutaneous silicone rubber implant with shallow surface microgrooves on the surrounding tissues in rabbits. Journal of Biomedical Materials Research 1997; 37: 539-547.

38 Parker JA, Walboomers XF, Von Den Hoff JW et al. Soft tissue response to microtextured silicone and poly-L-lactic acid implants: Fibronectin pre-coating vs. radio-frequency glow discharge treatment. Biomaterials 2002; 23: 3545-3553.

39 Picha GJ, Drake RF. Pillared-surface microstructure and soft-tissue implants: Effect of implant site and fixation. Journal of Biomedical Materials Research 1996; 30: 305-312.

40 von Recum AF, van Kooten TG. The influence of micro-topography on cellular response and the implications for silicone implants. Journal of Biomaterials Science. Polymer Edition 1995; 7: 181-198.

41 Kurnaz E, Kubaloglu A, Yilmaz Y et al. The effect of adjunctive Mitomycin C in Ahmed glaucoma valve implantation. European Journal of Ophthalmology 2005; 15 27-31.
42 Teixeira SH, Doi LM, De Freitas Silva AL et al. Silicone Ahmed glaucoma valve with and without intravitreal triamcinolone acetonide for neovascular glaucoma. Journal of Glaucoma 2012; 21: 342-348.

43 Costa VP, Azuara-Blanco A, Netland PA et al. Efficacy and safety of adjunctive mitomycin $C$ during Ahmed glaucoma valve implantation: A prospective randomized clinical trial. Ophthalmology 2004; 111: 1071-1076.

44 Ayyala RS, Zurakowski D, Smith JA et al. A clinical study of the Ahmed glaucoma valve implant in advanced glaucoma. Ophthalmology 1998; 105: 1968-1976.

45 Titze IR, Klemuk SA, Lu X. Adhesion of a monolayer of fibroblast cells to fibronectin under sonic vibrations in a bioreactor. Annals of Otology, Rhinology, and Laryngology 2012; 121: 364-374.

46 Ito Y, Kimura T, Ago Y et al. Nano-vibration effect on cell adhesion and its shape. Biomedical Materials and Engineering 2011; 21: 149-158.

47 Truskey GA, Pirone JS. The effect of fluid shear stress upon cell adhesion to fibronectintreated surfaces. Journal of Biomedical Materials Research 1990; 24: 1333-1353.

48 Vlaisavljevich E, Janka LP, Ong KG et al. Magnetoelastic materials as novel bioactive coatings for the control of cell adhesion. IEEE Transactions of Biomedical Engineering 2011; 58: 698-704.

49 Mackenzie PJ, Schertzer RM, Isbister CM. Comparison of silicone and polypropylene Ahmed glaucoma valves: Two-year follow-up. Canadian Journal of Ophthalmology 2007; 42: 227-232.

50 Electrostrictive and Magnetostrictive Systems. In: Katz HW, editor. Solid State Magnetic and Dielectric Devices. New York: John Wiley and Sons, 1959: 87-130.

51 Metglas Inc. Magnetic Alloy 2826MB (nickel-based) Technical Bulletin. Available at: http://www.metglas.com. (accessed at 26 April 2015).

52 Gutiérrez J, Lasheras A, Barandiarán JM et al. Improving the magnetoelectric response of laminates containing high temperature piezopolymers. IEEE Transactions on Magnetics 2013; 49: 42-45.

53 Liang C. Development of bulk-scale and thin film magnetostrictive sensor [PhD thesis]. Auburn, AL: Auburn University; 2007.

54 Masaki T, Kawata K, Masuzawa T. Micro electro-discharge machining and its applications. Proceedings of IEEE MEMS; 11-14 Feb 1990; Napa Valley, CA, USA; 1990: 21-26.

55 Friedman H. Photochemical machining. In: ASM Handbook, Volume 16: Machining. ASM International, 1989: 587-593.

56 Goldie JH, Gerver MJ, Oleksy J et al. Composite Terfenol-D sonar transducers. Proceedings of SPIE, Smart Structures and Materials 1999; 3675: 223-234.

57 Raskin D, Smith CH. Applications of amorphous metals: Progress and prospects. In: F. E. Luborsky, editor. Amorphous Metallic Alloys. London: Butterworths, 1983: 397.

58 Brouha $M$, van der Borst J. The effect of annealing conditions on the magnetomechanical properties of Fe-B-Si amorphous ribbons. Journal of Applied Physics 1979; 50: 7594-7596.

59 Livingston J, Morris W. SEM studies of magnetic domains in amorphous ribbons. IEEE Transactions on Magnetics 1981; 17: 2624-2626.

60 Hasegawa R, Narasimhan MC, DeCristofaro N. A high permeability Fe-Ni base glassy alloy containing Mo. Journal of Applied Physics 1978; 49: 1712-1714.

61 Torok E, Hausch G. Magnetoelastic effects of some ferromagnetic metallic glasses. Rapidly Quenched Metals III. Proceedings of the Third International Conference on Rapidly Quenched Metals; 3-7 Jul 1978; Brighton, England; 1978: 105-108.

62 Schmidt EM, Mclntosh JS, Bak MJ. Long-term implants of Parylene-C coated microelectrodes. Medical \& Biological Engineering \& Computing 1988; 26: 96-101.

63 Agarwala M, Bourell D, Beaman J et al. Direct selective laser sintering of metals. Rapid Prototyping Journal 1995; 1: 26-36.

64 DeCristofaro N. Amorphous metals in electric-power distribution applications. MRS Bulletin 1988; 23: 50-56.

65 Medical Electrical Equipment - Part 2-33: Particular Requirements for the Basic Safety and Essential Performance of MR Equipment for Medical Diagnosis, IEC Standard 606012-2-33, 2010.

66 Schmale I, Gleich B, Rahmer J et al. MPI safety in the view of MRI safety standards. IEEE Transactions on Magnetics 2015; 51: 18-21.

67 Koydemir HC, Kulah H, Ozgen C. Solvent compatibility of Parylene C film layer. Journal of Microelectromechanical Systems 2014; 23: 298-307.

68 Clark AE. Magnetostrictive rare-earth Fe2 compounds. In: Wohlfarth EP, editor. Ferromagnetic Materials, Volume 1. New York: North-Holland, 1980: 577.

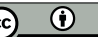

This work is licensed under a Creative Commons Attribution 4.0 Unported License. The images or other third party material in this article are included in the article's Creative Commons license, unless indicated otherwise in the credit line; if the material is not included under the Creative Commons license, users will need to obtain permission from the license holder to reproduce the material. To view a copy of this license, visit http://creativecommons. org/licenses/by/4.0 